\title{
Morin, quercetin, catechin and quercitrin as novel natural antimalarial candidates
}

\begin{abstract}
Background: The discovery of efficient antimalarial candidates from natural sources has become more urgent as a result of drug resistance emergence. Therefore, discovering effective substitutes to treat malaria remains a top priority to health community. Medicinal plants and some edible foods contain inexhaustible source of new active ingredients that may treat malaria.

Objective: The aim of the current study was to explore the in-vitro inhibitory effect of the polyphenolic flavonoids: morin, quercetin, catechin, and quercitrin on the $\beta$-hematin formation in order to utilize them as new potential antimalarial leads.

Methodology: The methods used were the in-vitro colorimetric semi-quantitative and quantitative assays that are based on $\beta$-hematin inhibition and optical density measurement at $405 \mathrm{~nm}$.

Results and discussion: The in-vitro inhibitory effect on $\beta$-hematin formation using the four flavonoids of morin, quercetin, catechin and quercitrin in comparison to chloroquine and amodiaquine positive controls signifies to their encouraging potential as new antimalarial leads. Morin flavonol however was the most potent compound even at extremely low concentration of $0.04 \mathrm{mg} / \mathrm{ml}$. The efficiency of the four flavonols suggesting the formation of a favorable complex with the free heme over $\beta$-hematin formation to a level that becomes irreversibly toxic to the plasmodium.
\end{abstract}

Keywords: anti-malaria, morin, quercetin, catechin, quercitrin, $\beta$-hematin
Volume 8 Issue 3 - 2020

Saleh Abu-Lafi,' Mutaz Akkawi, ${ }^{2}$ Fuad AlRimawi, ${ }^{3}$ Qassem Abu-Remeleh, ${ }^{2}$ Pierre Lutgen ${ }^{4}$

'Faculty of Pharmacy, Al-Quds University, Palestine ${ }^{2}$ Life Sciences Department, College of Science and Technology, Al-Quds University, Palestine

${ }^{3}$ Department of Chemistry, Faculty of Science and Technology, Al-Quds University, Palestine ${ }^{4}$ IFBV-BELHERB, Luxembourg

Correspondence: Mutaz Akkawi: Life Sciences Department College of Science and Technology, Al-Quds University, West Bank, Palestine, Phone ++972(0)526435785, Fax ++972-26260903, Email akkawi74@gmail.com

Received: May II, 2020 | Published: June 25, 2020

\section{Introduction}

The latest annual World Malaria Report 2019 statistics documented 228 million infected malaria cases worldwide associated with 405,000 fatalities. ${ }^{1}$ Despite the slow decline in mortality rates over the past decade, malaria remains the major cause of death among the most at-risk pregnant women and children in Africa. ${ }^{2}$ This is due in part to the growing antimalarial drug resistance towards Plasmodium falciparum, the most virulent parasite causing malaria. Therefore, discovering effective substitutes to treat malaria remains a top priority to the health professional community. Further studies are in need to develop new candidates from natural sources to combat the growing resistance of the currently used antimalarial drugs.

During the intraerythrocytic stage of the life cycle of malaria, plasmodium species degrade hemoglobin for their biosynthetic requirements, resulting in an accumulation of large amounts of monomeric ferriheme known as ferriprotoporphyrin (IX). It causes the generation of reactive oxygen species, which may induce oxidative stress leading to parasitic death. ${ }^{3-5}$

The parasite avoids these toxic effects by polymerizing these heme molecules within the food vacuole at $\mathrm{pH}$ 4.5-5.0, into a nontoxic, unreactive, insoluble detoxification by-product crystal called hemozoin or 'malaria pigment'. ${ }^{6,7}$ A synthetic analog to hemozoin, the $\beta$-hematin is structurally and spectroscopically identical to 'purified hemozoin' has been prepared and utilized making it an excellent target for in-vitro assay analysis. ${ }^{8-10}$ Antimalarial drugs are believed to act by inhibiting hemozoin formation and eventually killing the parasite. ${ }^{11}$

Medicinal plants and some edible foods are considered to be an inexhaustible source of active ingredients that were used for centuries to treat diseases. ${ }^{12,13}$ More attention was directed recently to various flavonoids present in fruits, vegetables and grains attributing to numerous biological activities. Flavonoids are a prominent class of polyphenolic phytonutrients that are responsible for the intense colors in flowers, fruits and vegetables. ${ }^{14}$ Flavonoids backbone consists of two phenyl rings $\mathrm{A}$ and $\mathrm{B}$ and a heterocyclic pyrone ring $\mathrm{C}$ (Figure 1). Variations in ring $\mathrm{C}$ substitutions result in several classes of flavonoids, including flavanols (or catechins), flavones, flavonols, flavonones and anthocyanidins. ${ }^{14}$ Within each class, there are still more subgroups, each of which carries its own health benefits. Sufficient studies have proven the role of flavonoids as effective antioxidants, ${ }^{15}$ antiinflammatory ${ }^{16}$ immunomodulatory ${ }^{17}$ and anticancer agents. ${ }^{18-20}$ The present work aimed to explore the in-vitro inhibitory effect of selected flavonoids, namely morin, quercetin, catechin, and quercitrin which are present in many fruits, vegetables and grains on the $\beta$-hematin formation using semi-quantitative and quantitative screening methods. This study is part of the continuing efforts to develop new alternative leads to combat antimalarial drug resistance.

\section{Materials and methods}

Dimethyl sulfoxide (DMSO), chloroquine diphosphate salt (CQ), amodiaquine hydrochloride (AD), morin, quercetin, gallic acid, catechin and quercitrin hydrate analytical standards, sodium acetate, and hemin chloride were all purchased from Sigma-Aldrich. Glacial acetic acid was purchased from Fluka. Ethanol (EtOH) was from Merck.

\section{In-vitro semi-quantitative method}

The method adopted was according to Deharo et al., ${ }^{21}$ assay with some modification..$^{22}$ A mixture containing $50 \mu \mathrm{L}$ of $0.5 \mathrm{mg} / \mathrm{mL}$ hemin 
chloride freshly dissolved in Dimethyl sulphoxide (DMSO), $100 \mu \mathrm{L}$ of $0.5 \mathrm{M}$ sodium acetate buffer $(\mathrm{pH} 4.4)$ and $50 \mu \mathrm{L}$ of the standard solution or either the negative (water, alkaline water, DMSO) or positive (chloroquine or amodiaquine) controls, was incubated in a non-sterile 96 -well flat bottom plate at $37^{\circ} \mathrm{C}$ for $18-24 \mathrm{~h}$. The solutions were added to the plate in the above order. The plate was then centrifuged for $10 \mathrm{~min}$ at $4000 \mathrm{rpm}$. The supernatant was removed and the $\mathrm{pH}$ of the reaction was measured. The final $\mathrm{pH}$ of the mixture was between (5.0-5.2). The wells were washed with $200 \mu \mathrm{L}$ DMSO per well to remove unreacted hemin. The plate was centrifuged again followed by discharging the supernatant. The $\beta$-hematin remaining was then dissolved in $200 \mu \mathrm{L}$ of $0.1 \mathrm{M} \mathrm{NaOH}$ to form an alkaline hematin that can be measured spectrophotometrically at $405 \mathrm{~nm}$ using ELISA reader. Positive controls were dissolved either in ultra-pure water (chloroquine) or DMSO (amodiaquine).

\section{Quantitative in-vitro method}

The adopted method was according to Blauer and Akkawi method. ${ }^{23,24}$ A freshly prepared stock solution of hemin chloride was prepared by dissolving the salt in DMSO and incubated for $30 \mathrm{~min}$ at $30^{\circ} \mathrm{C}$. Stock solutions of the standards used (morin or quercetin) were prepared also using alkaline water. $(0.5 \mathrm{M})$ sodium acetate buffer ( $\mathrm{pH} 4.4$ ) was also prepared, the final concentration of hemin in the reaction was $0.2 \mathrm{mM}$ and that of the morin and quercetin standards in the reaction was $0.4 \mathrm{mM}$, the whole mixture was left for $18-24 \mathrm{~h}$ at $37^{\circ} \mathrm{C}$ without stirring. The total volume of the reaction mixture was $32 \mathrm{ml}$, and the final $\mathrm{pH}$ was 4.9-5.2. Samples were centrifuged for 10 min using a serological (Jouan B4) centrifuge. The supernatant was discarded and the precipitate was washed with DMSO and quantitatively transferred to a Millipore Swinnex 13 filter containing Whatman filter paper No. 50, already lyophilized to a constant weight in a freeze-drying machine (Labconco Freezone). DMSO was passed slowly through the filter until the filtrate remained feebly colored and washed again with ultra-pure water. The remaining was then lyophilized to a constant weight.

\section{Results and discussion}

Pure morin, quercetin, catechin and quercitrin hydrate standards were examined individually to explore their potential as natural inhibitors for $\beta$-hematin formation using semi-quantitative and quantitative assays. Morin, quercetin and quercitrin are pigments that belong to flavonol class of flavonoids while catechin is categorized under flavanol class. Figure 1 shows the chemical structures of these pure standards examined in the current study. The absorption due to the remaining $\beta$-hematin in the wells was recorded at $405 \mathrm{~nm}$ as in figures 2-6.<smiles>O=c1c(O)c(-c2ccc(O)cc2O)oc2cc(O)cc(O)c12</smiles>

Morin<smiles>OC1=C(c2ccc(O)c(O)c2)Oc2cc(O)cc(O)c2C1</smiles>

Catechin<smiles>O=C(O)c1cc(O)c(O)c(O)c1</smiles>

Gallic acid<smiles>O=c1c(O)c(-c2ccc(O)c(O)c2)oc2cc(O)cc(O)c12</smiles>

Quercetin<smiles>C[C@@H]1O[C@@H](Oc2cc(-c3oc4cc(O)cc(O)c4c(=O)c3O)ccc2O)[C@H](O)[C@H](O)[C@@H]1O</smiles>

Quercitrin

Figure I Chemical structures of standards studied. 


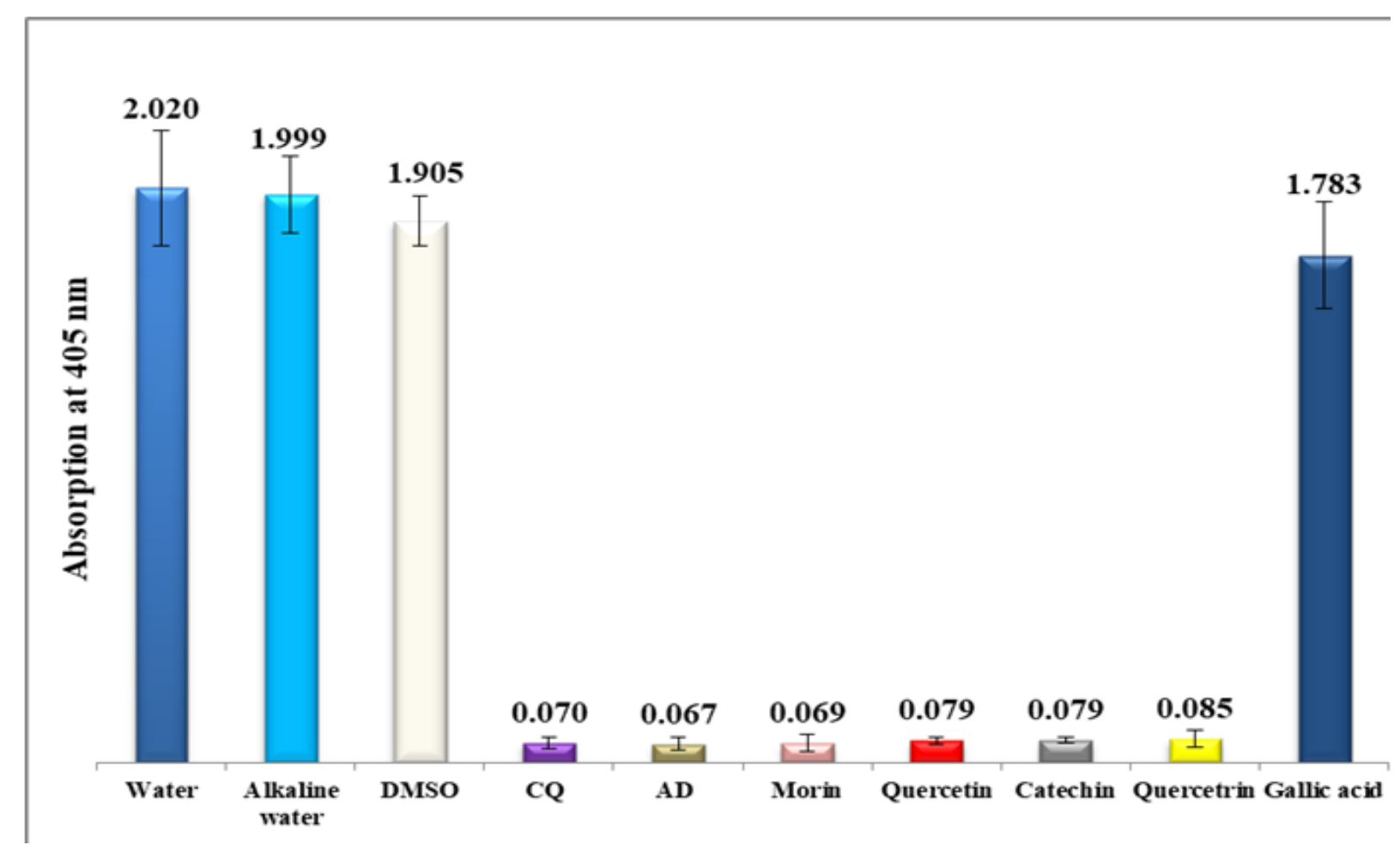

Figure 2 Column diagram representing the efficacy of four analytical standards (morin, quercetin, catechin and quercetrin) dissolved in alkaline water (all at $0.1 \mathrm{mg} / \mathrm{ml}$ ) compared to gallic acid dissolved in water at $1.0 \mathrm{mg} / \mathrm{ml}$, negative controls (water, DMSO and alkaline water) and positive controls (chloroquine CQ and amodiaquine $A D$, both at $0.1 \mathrm{mg} / \mathrm{ml}$ ). The absorption values of the dissolved $\beta$-hematin (alkaline hematin) were monitored at $405 \mathrm{~nm}$ using ELISA reader. Each result represents the average of 16 individual experiments.

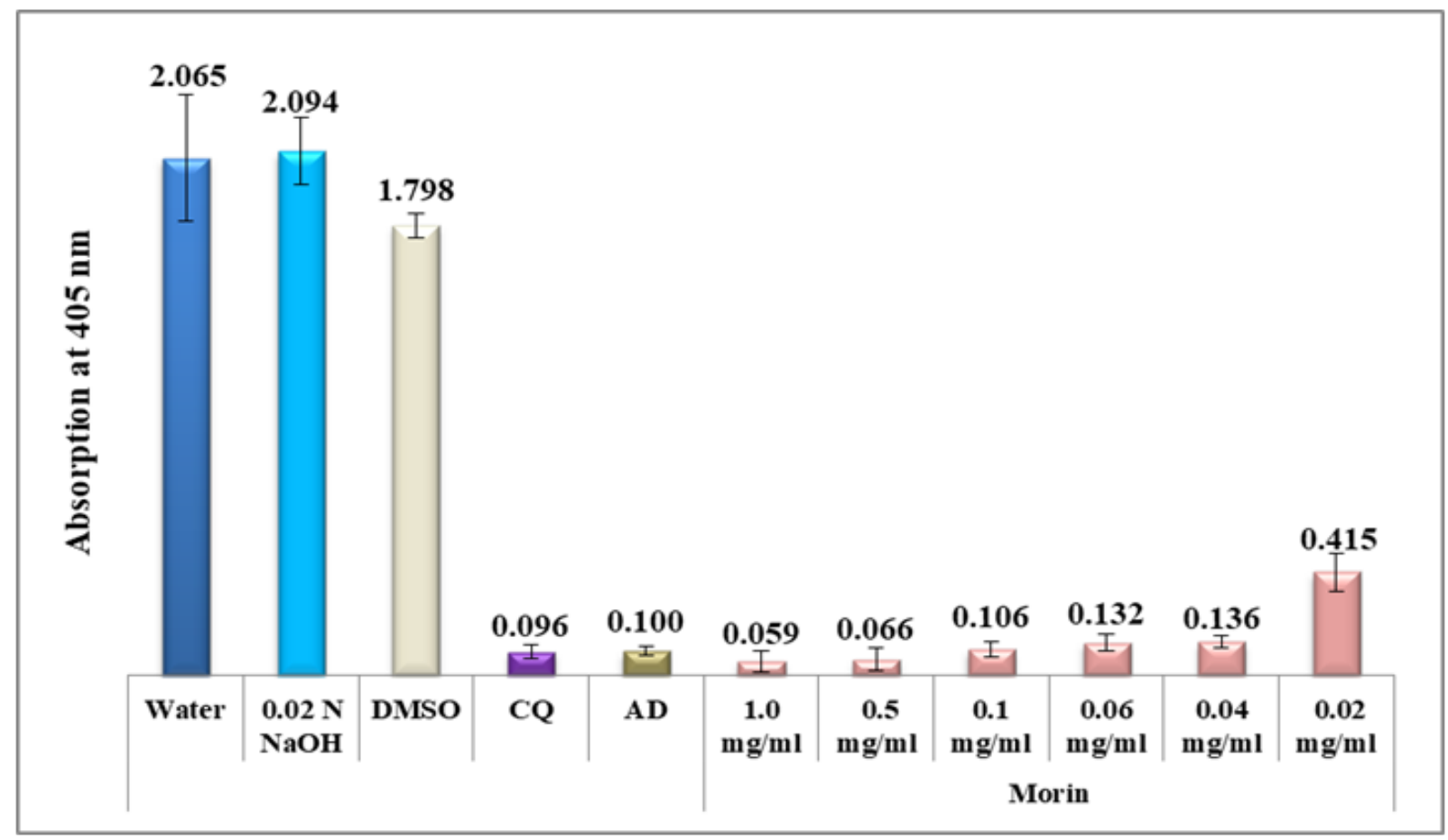

Figure 3 Column diagram representing the efficacy of morin analytical standard dissolved in alkaline water compared to the negative controls, water, DMSO and $0.02 \mathrm{~N} \mathrm{NaOH}$ and to positive controls, chloroquine (CQ) and amodiaquine (AD) both at $0.1 \mathrm{mg} / \mathrm{ml}$, showing the absorption values of dissolved $\beta$-hematin (alkaline hematin) at $405 \mathrm{~nm}$ using ELISA reader. Each result represents the average of 16 individual experiments. 


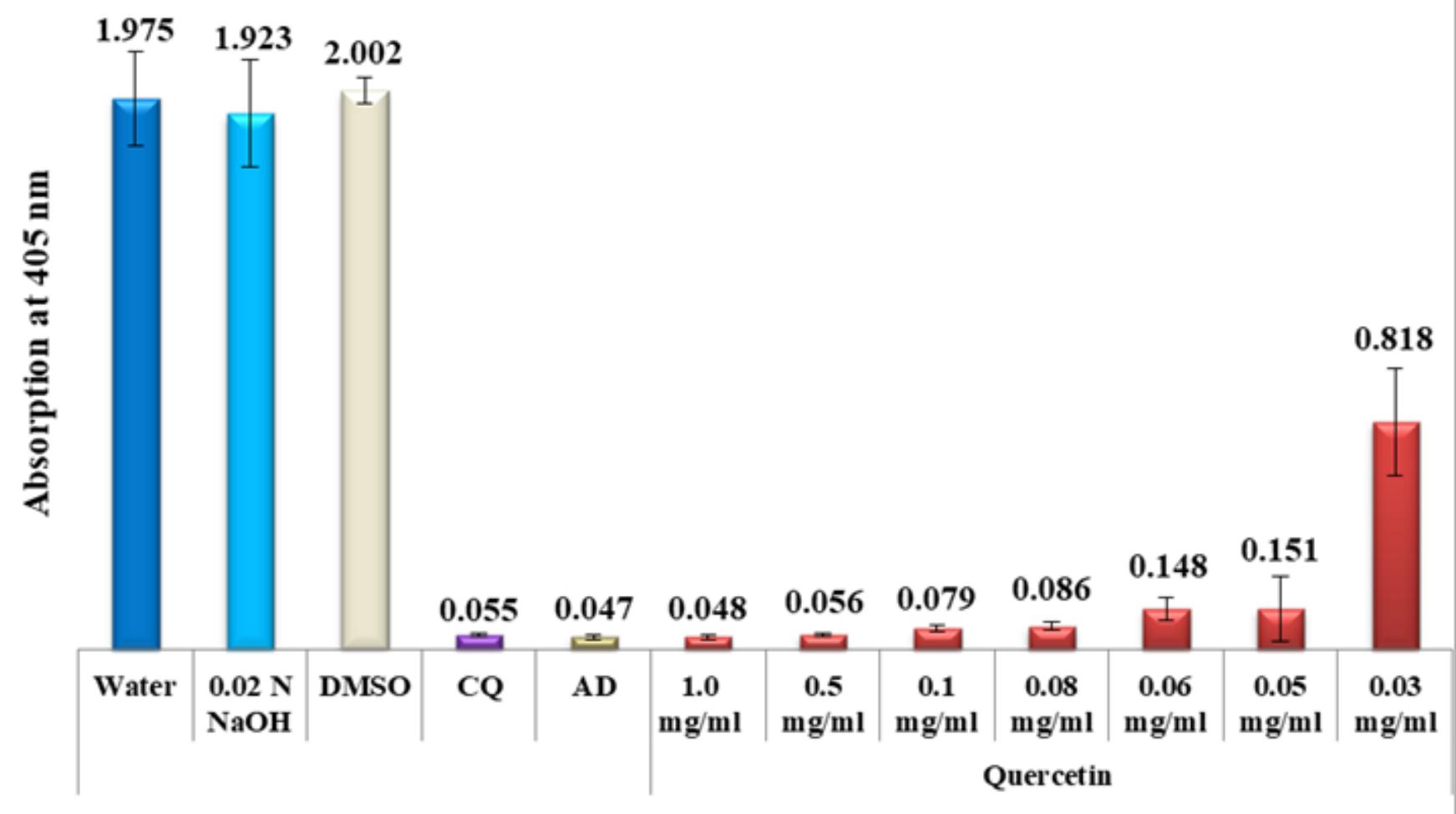

Figure 4 Column diagram representing the efficacy of quercetin analytical standard dissolved in alkaline water compared to the negative controls, water, DMSO and $0.02 \mathrm{~N} \mathrm{NaOH}$ and to positive controls, chloroquine (CQ) and amodiaquine (AD) both at $0.1 \mathrm{mg} / \mathrm{ml}$, showing the absorption values of dissolved $\beta$-hematin (alkaline hematin) at $405 \mathrm{~nm}$ using ELISA reader. Each result represents the average of 16 individual experiments.

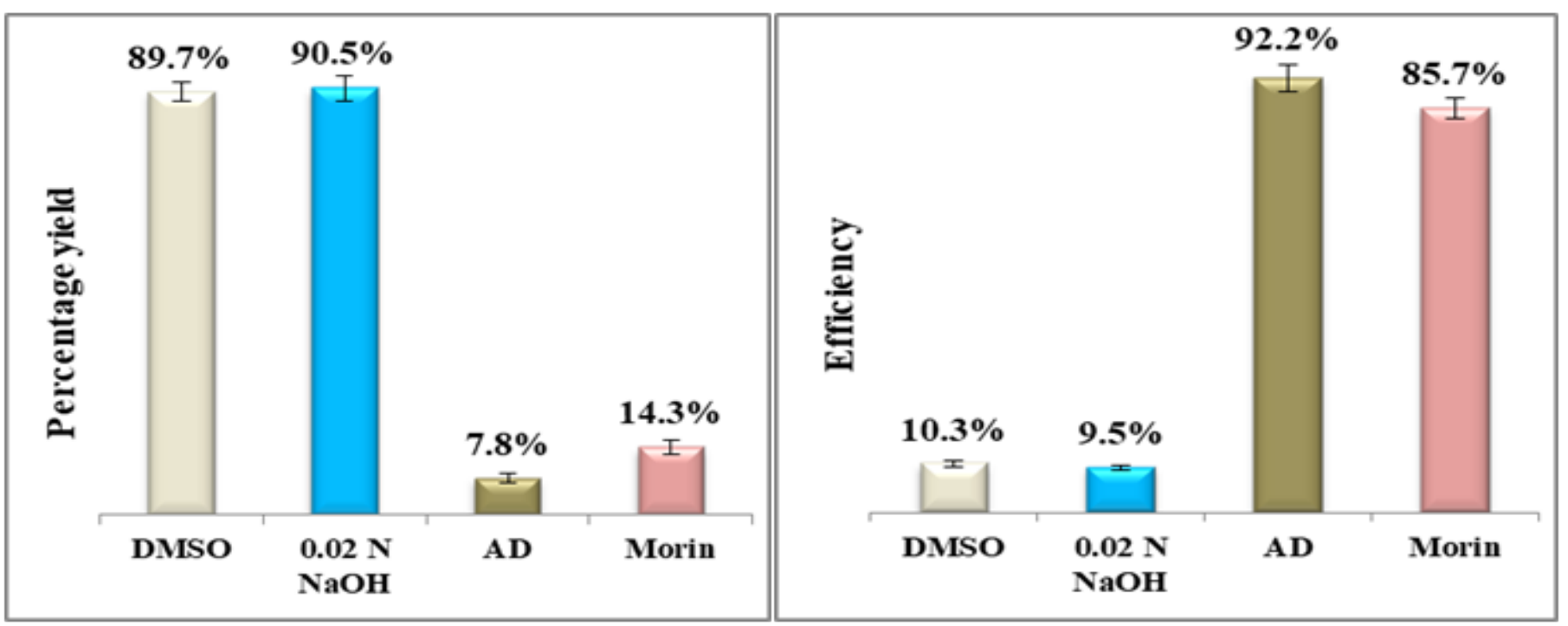

Figure 5 Column diagram of percentage yields of morin (A) and its efficiency (B) vs the positive control, amodiaquine (AD) both at $0.4 \mathrm{mM}$. Yields are inversely proportional to drug efficiency, the lower the percentage yield, the better the activity. 

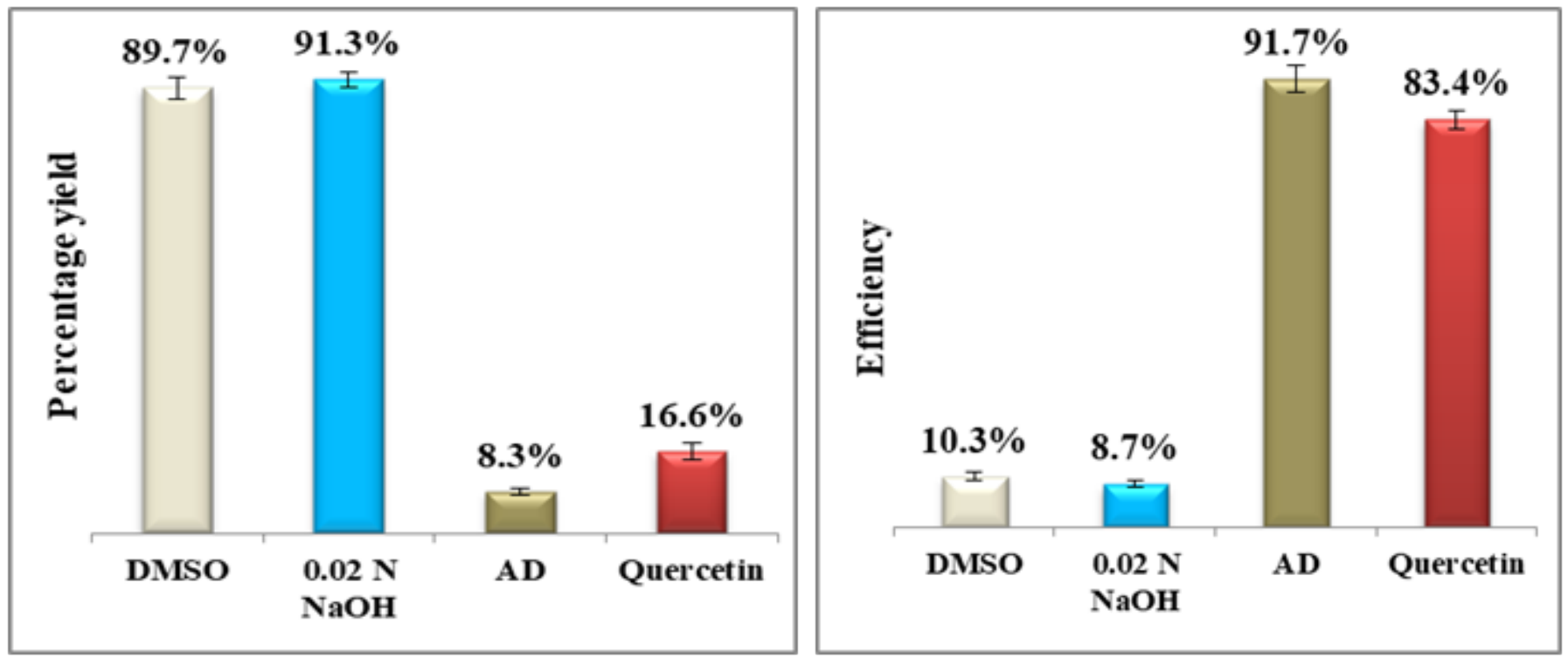

Figure 6 Column diagram of percentage yields of quercetin (A) and its efficiency (B) vs the positive control, amodiaquine (AD) both at $0.4 \mathrm{mM}$. Yields are inversely proportional to drug efficiency, the lower the percentage yield, the better the activity.

\section{Semi-quantitative method}

To combat the growing resistances of the currently used antimalarial drugs, the inhibitory effect of each standard of morin, quercetin, catechin, quercitrin hydrate and gallic acid on the bio-mineralization of ferriprotoporphyrin IX to $\beta$-hematin was investigated using a semi-quantitative microassay. ${ }^{21}$ Water, alkaline water and DMSO were used as negative controls giving rise to maximum absorption values. The spectrophotometric absorption values at $405 \mathrm{~nm}$ of each of the four standards (morin, quercetin, catechin and quercitrin) fixed at a concentration of $0.1 \mathrm{mg} / \mathrm{mL}$ ranged from 0.069 to 0.085 in comparison to 0.07 and 0.067 of chloroquine and amodiaquine positive controls respectively (figure 2), also it is important to note that gallic acid was inactive at a much higher concentration of $1.0 \mathrm{mg} / \mathrm{ml}$ with an absorption value of 1.783 . This encouraging result signifies the potential of flavonoids understudy in inhibiting $\beta$-hematin formation in-vitro. Morin proves to be the most potent among the four antimalarial examined candidates to a level similar to both chloroquine and amodiaquine positive controls (figure 2). This is an important result since it could pave the way to new alternative leads to combat malaria in the future. To further investigate the effect of concentration on their antimalarial potential, two concentrations below $0.1 \mathrm{mg} / \mathrm{ml}$ were prepared and tested in-vitro for all the four standards. As in table 1, morin remains the most effective flavonol suggesting the formation of a stable complex between free heme and morin over $\beta$-hematin to a level that becomes irreversibly toxic to the plasmodium. Morin remains active even at threshold down to 0.04 $\mathrm{mg} / \mathrm{ml}$ level.

Figure 3 shows a column diagram representing the efficacy of all concentrations of morin dissolved in $0.02 \mathrm{~N} \mathrm{NaOH}$ compared to two positive and three negative controls. Apparently, not only the use of alkaline solution increased morin solubility, but it also facilitated the $\beta$-hematin inhibition by favoring the formation of a stable complex with free heme. It is noteworthy to note that the trihydroxy benzoic acid, gallic acid, disclosed no effect (table 1).
.Quercetin and morin are isomers, with a catechol-type B-ring in quercetin and a resorcinol-like $\mathrm{B}$ ring in morin. Both isomers show strong activity in inhibiting the formation of $\beta$-hematin, with quercetin to a lesser extent (table 1, figures $3 \& 4$ ).

Table I Absorbance of four standards dissolved in alkaline water at concentrations $<0.1 \mathrm{mg} / \mathrm{ml}$ compared to gallic acid, dissolved in water at a higher concentration

\begin{tabular}{lll}
\hline Standard & Concentration $(\mathbf{m g} / \mathrm{ml})$ & Absorbance at $\mathbf{4 0 5} \mathbf{~ n m}$ \\
\hline Morin & 0.04 & 0.136 \\
& 0.02 & 0.415 \\
Quercetin & 0.05 & 0.151 \\
& 0.03 & 0.818 \\
Catechin & 0.06 & 0.133 \\
& 0.04 & 1.409 \\
Quercitrin & 0.06 & 0.511 \\
& 0.04 & 1.685 \\
Gallic acid & 1 & 1.783 \\
& 0.5 & 1.861 \\
\hline
\end{tabular}

Moreover, quercitrin, a glycoside formed from quercetin backbone and rhamnose deoxy sugar also shows a significant activity and to a greater extent the catechin standard which lack carbonyl on its ring (figure 1, table 1).

\section{Quantitative method}

Figure 5 shows a comparison in terms of percentage yield vs efficiency of morin using the quantitative assay method. ${ }^{23,24}$

Results display a considerable potential of morin with a percentage yield of $\beta$-hematin formation of $14.3 \%$ and an efficiency of $85.7 \%$ compared to $92.2 \%$ using amodiaquine positive control. Therefore, 
it may be considered as a potential practical antimalarial substitute to currently used drugs suffering resistance since the in-vitro and invivo studies proved that morin exhibits very low toxicity levels and its chronic administration is well tolerated. ${ }^{25}$ For quercetin, however, the percentage yield of $\beta$-hematin formation was $16.6 \%$ compared to $8.3 \%$ of amodiaquine positive control (figure 6), while quercetin efficiency was $83.4 \%$ vs $91.7 \%$ for amodiaquine positive control. It is important to note that flavonoids may have a synergistic effect when used in combination with artemisinin. ${ }^{26}$

All the accumulated semi-quantitative and quantitative results encourage us to investigate more flavonoids and their effect as $\beta$-hematin inhibitors in comparison to currently in use antimalarial drugs.

\section{Flavonoid-heme complex formation}

It is believed that the mechanism of $\beta$-hematin inhibition happens via the formation of a stable complex comprising the antimalarial drug and ferriheme. ${ }^{22}$ It is still however a challenging task to extrapolate the exact mechanism of such a stable complex. There are three interaction forces that are probably involved in the formation of [flavonoid-heme] complex. The most important interaction that plays a key role is the $\mathrm{H}$-bonding between the free propionic carboxylate heme moieties with the hydroxychromone groups of the flavonoids particularly ring B and C (figure 1). This is important because gallic acid possessing only one ring with three hydroxyl groups was inactive. Another question may arise at this point of what is the stoichiometric ratio between flavonoid and heme in this complex. Spectrophotometric titration may give insight to such a rational question. Furthermore, the hydrophobic $\pi-\pi$ stacking between flavonoid rings and porphyrin rings may also play a significant role in the mechanism. Finally the binding of the flavonoid ketonic and/or the hydroxyl sites with the iron may play a minor role in such a mechanism. However, there may be two possible chelating sites with ring $\mathrm{B}$ and $\mathrm{C}$ in competition. Keeping in mind the acidic $\mathrm{pH}$ used in the experiments, most probably a di-ketone may be formed on ring B making it less affordable for binding with the heme. ${ }^{27}$ Identification of flavonoid-heme complex using molecular modeling with appropriate docking fit studies can give answers to such binding related questions which remains a challenge for further investigation.

\section{Conclusion}

With the emerging resistance of the currently used antimalarial drugs either as pure active compounds or as in combination, there is still a continuous need for the development of new leads. In this effort, flavonoids such as morin and quercetin showed promising antimalarial activity compared to chloroquine and amodiaquine drugs in-vitro. Flavonoids are present in fruits and vegetables in high quantity which make it more favorable cheap, available alternative candidates over synthetic antimalarial drugs which by time have developed resistance. Using a homologous series of flavonoids may lead to more promising antimalarials substitutes.

\section{Acknowledgments}

None.

\section{Conflicts of interest}

The authors declare that there is no conflict of interest.

\section{References}

1. World Malaria Report. World Health Organization; 2019.

2. UN News. Malaria: Focus on pregnant women and children, stresses UN health agency report; 2019.

3. Kumar S, Guha M, Choubey V, et al. Antimalarial Drugs Inhibiting Hemozoin (Beta-Hematin) Formation: A Mechanistic Update. Life Sciences. 2007;80(9):813-828.

4. Rosenthal PJ. Cysteine proteases of malaria parasites. Int $J$ Parasitol. 2004;34(13-14):1489-1499.

5. Tripathy S, Roy S. Redox sensing and signaling by malaria parasite in vertebrate host. J Basic Microbiol. 2015;55(9):1053-1063.

6. Goldberg D, Slater A, Cerami A. et al. Hemoglobin degradation in the malaria parasite Plasmodium falciparum: An ordered process in a unique organelle. Proc Natl Acad Sci USA. 1990;87(8):931-935.

7. Akkawi M, Jaber S, Abu-Laf S. et al. HPLC separation and in vitro antimalarial studies of Artemisia annua plants from two different origins: Cameroon versus Luxembourg. MWJ. 2014;5:11.

8. Slater GA, Swiggard WJ, Orton BR, et al. An Iron-Carboxylate Bond Links the Heme Units of Malaria Pigments. Proc Natl Acad Sci USA 1991;15:88(2):325-329.

9. Pagola S, Stephens PW, Bohle SD, et al. The structure of malaria pigment $\beta$-haematin. Nature. 2000;404(6775):307-310.

10. Akkawi M, Jaber S, Abu-Remeleh Q, et al. Investigations of Artemisia Annua and Artemisia Sieberi Water Extracts Inhibitory Effects on $\beta$-Hematin Formation. Med Aromat Plants. 2014;3:150.

11. Tekwani B, Walker L. Targeting the Hemozoin Synthesis Pathway for New Antimalarial Drug Discovery: Technologies for In Vitro $\beta$-Hematin Formation Assay. Comb Chem High Throughput Screen. 2005;8(1):6379.

12. Seo J, Lee S, Elam M, et al. Study to find the best extraction solvent for use with guava leaves (Psidium guajava L.) for high antioxidant efficacy. Food Sci Nutr. 2014;2(2):174-180.

13. Gutierrez RM, Mitchell S, Solis RV. Psidium guajava: a review of its traditional uses, phytochemistry and pharmacology. J Ethnopharmacol. 2008;117:1-27.

14. Ahn-Jarvis J, Parihar A, Doseff A. Dietary Flavonoids for Immunoregulation and Cancer: Food Design for Targeting Disease. Antioxidants. 2019;8(7):202.

15. Agati G, Azzarello E, Pollastri S, et al. Flavonoids as antioxidants in plants: Location and functional significance. Plant Science. 2012;196:67-76.

16. Ginwala R, Bhavsar R, Chigbu DG, et al. Potential Role of Flavonoids in Treating Chronic Inflammatory Diseases with a Special Focus on the AntiInflammatory Activity of Apigenin. Antioxidants. 2019;8(2):35.

17. Hosseinzade A, Sadeghi O, Biregani A, et al. Immunomodulatory Effects of Flavonoids: Possible Induction of T CD4+ Regulatory Cells Through Suppression of mTOR Pathway Signaling Activity. Front Immunol. 2019;10:51.

18. Kopustinskiene D, Jakstas V, Savickas, et al. Flavonoids as Anticancer Agents. Nutrients. 2020;12(2):457.

19. Liu HL, Jiang WB, Xie MX. Flavonoids: Recent Advances as Anticancer Drugs. Recent Pat Anticancer Drug Discov. 2010;5(2):152-164.

20. Chahar M, Sharma N, Dobhal M, et al. Flavonoids: A versatile source of anticancer drugs. Pharmacogn Rev. 2011;5(9):1-12.

21. Deharo E, Garcia RN, Oporto P, et al. A non-radiolabelled ferriprotoporphyrin biomineralisation inhibition test for the high throughput screening of antimalarial compounds. Exp Parasitol. 2002;100(4):252-256. 
22. Abu-Lafi S, Akkawi M, Abu-Remeleh Q, et al. Curcumin, a natural isolate from Curcuma longa (turmeric) with high $\beta$-hematin inhibitory potential. Pharm Pharmacol Int J. 2019;7(1):22-26.

23. Blauer G, Akkawi M. Investigations of B and $\beta$-hematin. J Inorg Biochem. 1997;66(2):145-152.

24. Blauer G, Akkawi M. Alcohol-water as a novel medium for beta-hematin preparation. Arch Biochem Biophys. 2002;398(1):7-11.

25. Caselli A, Cirri P, Santi A, et al. Morin: A Promising Natural Drug. Curr Med Chem. 2016;23(8):774-791.
26. Weathers PJ, Reed K, Hassanali A, e al. Chapter 4: Whole plant approaches to therapeutic use of Artemisia annua, L. (Asteraceae). IN: Artemisia annua. Pharmacology and Biotechnology. Eds., T Aftab, JFS Ferreira, MMA Khan, M Naeem Springer: Heidelberg, GDR; 2013 51-74 p.

27. Yang L, Li P, GAO YJ, et al. Qualitative observation of chemical change rate for quercetin in basic medium characterized by time resolved UV-vis spectroscopy. Journal of Molecular Liquids. 2010;151(2-3):134-137. 